\title{
Dinámica de la inversión extranjera directa en los estados de México: un análisis de cadenas de Markov espaciales
}

\author{
Dynamics of foreign direct investment in the states of Mexico: a Spatial \\ Markov chain analysis \\ Víctor Hugo Torres Preciado*, Mayrén Polanco Gaytán \\ y Miguel A. Tinoco Zermeño
}

Universidad de Colima, México

Recibido el 22 de enero de 2015; aceptado el 14 de septiembre de 2015

Disponible en Internet el 20 de septiembre de 2016

\section{Resumen}

El objetivo de esta investigación consiste en analizar la evolución de la distribución espacial y temporal de la inversión extranjera directa (IED) en las entidades federativas de México. La literatura que aborda el análisis de la IED en México es abundante y diversa; sin embargo, se argumenta que el análisis de la distribución espacio-temporal de la IED condicionada a la interacción espacial en México, aún está ausente. En este sentido, mediante la aplicación del enfoque de cadenas de Markov espaciales propuesto por Rey (2001), se encuentra que la divergencia regional en la captación de IED es un proceso que parece afianzarse cuando se analizan diferentes cortes en el tiempo. En particular, durante el periodo entre 2006 y 2013 el proceso de divergencia hacia estratos de mayor captación estaría impulsado por las entidades federativas que interactúan con entidades contiguas ubicadas en estratos de captación de IED menores.

(C) 2016 Universidad Nacional Autónoma de México, Facultad de Contaduría y Administración. Este es un artículo Open Access bajo la licencia CC BY-NC-ND (http://creativecommons.org/licenses/by-nc-nd/4.0/).

Códigos JEL: F21; R12; C46

Palabras clave: Inversión extranjera directa regional; Cadenas de Markov espaciales; México

\footnotetext{
* Autor para correspondencia.

Correo electrónico: torrespreciado@ucol.mx (V.H. Torres-Preciado).

La revisión por pares es responsabilidad de la Universidad Nacional Autónoma de México.
} 


\begin{abstract}
The aim of this investigation is to analyze the evolution of the spatio-temporal distribution of foreign direct investment (FDI) across Mexican states. The literature that analizes foreign direct invesment in Mexico is numerous and diverse; however, it is argued that the analysis of the spatio-temporal distribution of FDI conditioned to spatial interaction effects in Mexico, is still absence. In this sense, by applying the spatial Markov chain approach as proposed by Rey (2001), we found a divergence process in the FDI inflows among mexican states that seems to get stronger over time. In particular, during the period from 2006 to 2013, the process of divergence towards higher-FDI-inflows quantiles occurs among states spatially associated with neighbors in lower-FDI-quantiles.

(C) 2016 Universidad Nacional Autónoma de México, Facultad de Contaduría y Administración. This is an open access article under the CC BY-NC-ND license (http://creativecommons.org/licenses/by-nc-nd/4.0/).
\end{abstract}

JEL classification: $\mathrm{F} 21 ; \mathrm{R} 12 ; \mathrm{C} 46$

Keywords: Foreign direct investment; Spatial Markov chains; México

\title{
Introducción
}

En el caso de México, diversas reformas económicas y sus consecuentes cambios legislativos han sido implementados con la finalidad de promover la atracción de inversión extranjera directa hacia el país. Aunque las políticas implementadas durante las últimas décadas del siglo XIX que permitieron la participación en sectores económicos como la minería, los textiles, el transporte e incluso la electricidad y la banca, condujeron a incrementar significativamente la infraestructura disponible en el territorio, el principal cambio que impulsó un flujo importante de inversión extranjera directa ocurrió con la implementación del TLCAN y las modificaciones a la ley de inversión extranjera que le acompañaron (Dussel, 2000). A partir del TLCAN, el gobierno mexicano ha seguido diferentes estrategias para promover la liberalización del comercio y favorecer la atracción de los flujos de inversión extranjera; ellas incluyen los nuevos acuerdos y tratados de libre comercio como el firmado en el año 2000 con la Unión Europea (TLCUE), la suscripción de los Acuerdos para la Promoción y Protección Recíproca de las Inversiones, así como las mejorías en materia de trámites y transparencia.

En el ámbito regional, una modificación legislativa con implicaciones relevantes para el progreso económico de las entidades federativas consistió en permitir que la inversión extranjera directa pudiera ubicarse en cualquier región del país. Esta modificación, que vino a erradicar las barreras de tipo geográfico a la ubicación de la inversión extranjera, también significó un acceso equitativo a este tipo de inversión por parte de los diferentes estados del país, situación que ha derivado en un incremento en la competencia regional por atraer la inversión extranjera directa (IED) y reenfocar los esfuerzos de los gobiernos estatales en la implementación de políticas regionales para su atracción. No obstante, algunos estados han sido más exitosos que otros respecto a su capacidad de atracción de inversión extranjera, por ejemplo, solamente el Distrito Federal y Nuevo León captaron el 60 por ciento de la IED acumulada entre 2009 y 2013.

En este contexto de esfuerzos gubernamentales por incrementar el flujo de IED hacia el país, diferentes investigaciones se han enfocado en analizar el efecto de la inversión extranjera directa en la economía mexicana. Al respecto, la evidencia empírica sugiere que la IED ejerce efectos positivos limitados, y que aún deben mejorarse las condiciones para aprovechar el potencial de 
la IED y revertir los efectos negativos (Dussel, Galindo, Loría y Mortimore, 2007). Por ejemplo, el trabajo de Oladipo y Vásquez (2009) estudia el efecto de la inversión extranjera directa en el crecimiento económico agregado de México. Sus resultados sugieren que el efecto es favorable y significativo, aunque menor en comparación con las exportaciones, atribuyendo un rol relevante al TLCAN. Por su parte, Waldkirch (2010) analiza el efecto de la IED en la productividad industrial y los salarios en los diez años posteriores al inicio del TLCAN. El autor encuentra un efecto positivo en la productividad factorial total pero un efecto negativo en los salarios, lo que indica una divergencia entre ambos. En otro estudio, Mendoza (2011) evalúa el impacto de la inversión extranjera directa en el crecimiento del sector manufacturero en México, hallando evidencia de un efecto positivo y significativo de la IED únicamente cuando se considera simultáneamente el efecto del personal calificado.

Aunque estos estudios confirman la importancia que la inversión extranjera directa tiene para el progreso económico de México, en un contexto de concentración regional de la inversión extranjera directa, se torna relevante analizar su comportamiento espacial y temporal. El análisis de su evolución permitirá determinar la presencia de alguna tendencia a la convergencia o dispersión en la captación de IED, la expectativa que tienen los estados para transitar hacia niveles mayores (o menores) de captación de IED, e incluso si la interacción espacial con los estados vecinos está vinculada con esta transición.

El objetivo de esta investigación consiste en realizar un análisis acerca de la evolución de la distribución espacial y temporal de la inversión extranjera directa en las entidades federativas de México. La metodología que se propone para abordar este objetivo se basa en el enfoque de cadenas de Markov, en particular el enfoque propuesto por Rey (2001), que permite incorporar la interacción espacial entre regiones ${ }^{1}$. A diferencia de las metodologías que estudian la dinámica regional a partir de los enfoques de convergencia beta que ofrecen un panorama limitado acerca del comportamiento global de la distribución temporal de variables económicas (Quah, 1993) y del movimiento de las unidades espaciales dentro de la distribución (Rey, 2001), el enfoque de cadenas de Markov facilita el estudio de la dinámica de la distribución de las variables económicas a través del tiempo (Quah, 1996) y posibilita incluir la dimensión espacial en el análisis (Rey, 2001).

El documento se organiza de la siguiente manera: después de establecer el propósito de la investigación, en la primera sección se ofrece una revisión de la literatura; posteriormente, en la segunda sección se aborda el panorama regional de la inversión extranjera directa en México; después, en la tercera sección se explica la metodología a aplicar; enseguida, en la cuarta sección se analiza la dinámica de la distribución espacio-temporal de la inversión extranjera directa en México; y finalmente, se presentan las conclusiones.

\section{Revisión de la literatura}

La permanente adecuación del marco legislativo nacional con la finalidad de promover la inversión extranjera directa en México responde a la importancia que se le atribuye como factor promotor del progreso económico, la creación de empleo, la difusión de conocimiento tecnológico hacia el sector productivo nacional, nuevas formas de organización empresarial, la formación de capital humano especializado, la expansión del comercio internacional, la integración comercial

\footnotetext{
${ }^{1}$ En esta investigación se considera como región a una entidad federativa, de tal manera que en el texto se utilizan indistintamente ambos términos.
} 
con la industria local y como estabilizador macroeconómico al favorecer el ingreso de divisas al país (Dussel, 2000), (UNCTAD Secretariat, 2003). Sin embargo, algunos estudios establecen que el conjunto de beneficios obtenidos como resultado de la captación de IED no aparecen de forma automática, incluso en países donde se establecen requisitos de desempeño a los inversionistas extranjeros (Kumar, 2003).

En este sentido, aunque las investigaciones que abordan la IED en el país son numerosas, es posible identificar dos vertientes que predominan en el análisis. Por un lado, se encuentran los estudios que abordan el impacto que la IED ha tenido en el desempeño de la economía mexicana, y por otro, aquellas investigaciones cuya finalidad es conocer los factores que determinan la capacidad de atracción y localización de IED en el país. Los resultados de las investigaciones distan de alcanzar un consenso que apunte hacia la presencia indiscutible de efectos favorables para el progreso económico del país; de hecho, en algunos casos los resultados son poco concluyentes, y en otros, los efectos positivos son limitados e incluso negativos.

Por ejemplo, en un estudio elaborado por Oladipo (2007), el autor investiga el efecto de la IED en el crecimiento económico de México antes y después del TLCAN, sus resultados encuentran evidencia de un efecto positivo aunque también sostiene que el efecto de las exportaciones es comparativamente mayor. Estos resultados son confirmados posteriormente en el trabajo elaborado por Oladipo y Vásquez (2009). En otro estudio, de la Cruz, Canfield y González (2009) también encuentran evidencia de un efecto positivo por parte de la IED en el crecimiento económico de México, sin embargo, los autores sostienen que este efecto se observa principalmente en el crecimiento de la producción industrial e incidentalmente en el crecimiento de la producción en el sector servicios. Asimismo, encuentran que la IED también ejerce un efecto favorable como promotor del comercio internacional al observarse un efecto significativo en las exportaciones. En un trabajo más reciente, Romero $(2012,2014)$ encuentra que el efecto del capital extranjero en el crecimiento económico de México entre 1984 y 2011 es reducido y menor que el capital privado nacional, quizá por la débil presencia de encadenamientos de la IED con la economía nacional, lo cual sugiere que el capital extranjero debe ser considerado como complementario a la inversión privada nacional.

Por otro lado, diversos estudios han contribuido a esclarecer la importancia de la IED en el ámbito regional y sectorial. Un estudio pionero en este sentido, es el elaborado por Mejía (2005) quien destaca la asociación positiva entre una medida del potencial de mercado y el flujo de IED hacia las entidades del país. Posteriormente, Díaz-Bautista (2006) encuentra que algunas variables como los costos de transporte y migración hacia Estados Unidos son significativos para explicar el crecimiento económico regional de México, aunque también encuentra que la IED y el capital humano parecen no influir significativamente en el progreso económico de las regiones. En otro estudio, Mendoza (2011) estima el efecto de la IED sobre el crecimiento en nueve subsectores manufactureros de México, y mediante la aplicación de un modelo de panel, encuentra que el efecto de la IED es poco concluyente hasta que se considera la influencia del personal calificado.

Entre las investigaciones que abordan el efecto de la IED en el mercado laboral mexicano se encuentra el elaborado por Ernst, Berg y Auer (2007) quienes señalan que la IED ha tenido efectos favorables en la creación de empleo en el país, aunque esta se ha focalizado en la industria maquiladora de exportación. Los autores señalan que los salarios en este sector han observado un incremento aunque menor al promedio del sector manufacturero. Por su parte, Waldkirch (2010) aborda el efecto de la IED en la productividad total de factores y en el mercado laboral. El autor señala que en los primeros diez años posteriores a la puesta en marcha del TLCAN, la IED ha ejercido un efecto favorable en la dinámica de la productividad total de factores nacional, aunque 
también ha encontrado evidencia de un efecto negativo en los salarios particularmente de la IED que proviene de Estados Unidos hacia la industria no maquiladora. En otro estudio, Airola (2008), quien utiliza los datos de la Encuesta Nacional de Ingreso y Gasto de los Hogares para varios años, señala que la IED favorezca el aumento de los salarios relativos incluso para los trabajadores más educados, y por tanto, la dispersión salarial en México. Recientemente, Vergara, de Jesús y Carbajal (2015) analizan el efecto de la IED por gran división en los estados de la frontera norte, entre sus principales hallazgos destaca la presencia de un efecto no significativo en la creación de empleo, con excepción del sector eléctrico.

Por otro lado, algunas investigaciones se han enfocado en evaluar la capacidad de la IED para facilitar la difusión de conocimiento tecnológico y organización hacia las empresas nacionales. En este aspecto, Romo (2003) encuentra que la IED establecida en México ha contribuido al desarrollo de capacidades tecnológicas a través de efectos de demostración y entrenamiento de capital humano, pero de forma no significativa mediante la colaboración con empresas nacionales. En otro estudio, Domínguez y Brown (2004) encuentran para una muestra de establecimientos con capacidades tecnológicas heterogéneas, que la difusión de conocimiento tecnológico de la IED hacia las empresas nacionales no es significativo. Sin embargo, cuando los autores segmentan la muestra por nivel de capacidades tecnológicas, encuentran que este efecto es positivo y significativo en el segmento con alto nivel capacidades tecnológicas.

Entre las investigaciones que analizan los factores que determinan la capacidad de atracción y localización de IED en el país se encuentra el estudio elaborado por Cuevas, Messmacher y Werner (2005) quienes evalúan el impacto de los acuerdos de libre comercio en la IED para un conjunto de países, encuentran que el TLCAN favoreció la presencia de IED en México por un sesenta por ciento mayor a una situación en que no hubiera existido dicho tratado comercial. Dussel et al. (2007) abordan los determinantes macroeconómicos de la IED a escala sectorial y regional. Los autores señalan que la elasticidad de las materias primas importadas con respecto a la IED es positiva y negativa para las materias primas de origen nacional, lo cual sugiere una limitada capacidad de la industria nacional para abastecer a las transnacionales. Aspecto que implica a su vez un limitado proceso de difusión de conocimiento tecnológico en beneficio de la industria nacional. En un estudio con datos de panel, Juárez y Ángeles (2013) sostienen que la IED se establece principalmente en estados con un desarrollo comparativamente mayor que otros, y que el tamaño del mercado estatal continúa siendo relevante como factor de atracción. Por su parte, Escobar (2013) quien analiza los determinantes de la IED a escala estatal en México, encuentra que mayores niveles educativos y menores tasas de delincuencia favorecen la atracción de IED. También encuentra presencia de efectos de difusión interregionales, aspecto que parece indicar una situación complementariedad entre la IED que se establece en un estado específico y los estados contiguos.

En el estudio de la IED que llega a México, también se ha puesto atención en el rol de la industria maquiladora de exportación (IME). Aunque se ha documentado que la IME nació en la década de los sesenta como un programa de importación de insumos, equipo y maquinaria, entre otros factores de producción, para la elaboración de productos que serían exportados a Estados Unidos, con la finalidad de generar empleo (Vera-Cruz, Dutrénit y Gil, 2005), también se ha señalado que se trata más bien de un régimen arancelario (García, 2001), también se ha documento la evolución de la IME hacia estadios de mayor complejidad y desarrollo tecnológico, proceso en el que la IED ha tenido un rol preponderante (Carrillo, 2000). En este sentido, algunos estudios enfocados al análisis de casos particulares coinciden en señalar que si bien la IED inmersa en la IME ha logrado propiciar la difusión de capacidades tecnológicas y el desarrollo empresarial mediante la formación de capital humano emprendedor y cadenas de proveeduría, 
también ha evolucionado lentamente en integrarse al sistema productivo local (Carrillo, 2001), (Vera-Cruz et al., 2005).

En este contexto, los esfuerzos de investigación realizados han permitido avanzar en el conocimiento acerca del efecto e incluso los determinantes de la IED en México, cuyos resultados apuntan hacia la importancia de establecer políticas de atracción funcionales que prioricen la calidad y la integración de la IED con la economía nacional, buscando endogenizar sus beneficios potenciales y minimizar los efectos negativos (Dussel et al., 2007). No obstante este avance, aún es necesario profundizar en el análisis de la dinámica regional de la IED en México, en particular la evolución de su distribución espacial y temporal en las entidades federativas de México. En este sentido, hasta donde se tiene conocimiento, el estudio de la dinámica espacial y temporal de la IED mediante cadenas de Markov ha sido elaborado por Wren y Jones (2010) para las regiones británicas, aunque se realiza mediante el enfoque Markoviano clásico, sin considerar los posibles efectos de contigüidad espacial ${ }^{2}$.

\section{Panorama de la inversión extranjera directa en los estados de México}

La evolución reciente de los flujos de inversión extranjera directa a escala global ha mostrado fluctuaciones importantes relacionadas con el ambiente de fragilidad macroeconómica internacional y la incertidumbre en las políticas de inversión de los países receptores. En particular, ello sucedió después de experimentar una reducción del 18 por ciento en los flujos de IED globales durante 2012, comportamiento a su vez acompañado por un incremento en la participación de los países en desarrollo del 52 por ciento, (UNCTAD, 2013). No obstante, en 2013 hubo una recuperación del 9 por ciento en el flujo mundial de inversión.

En el caso de México, la evolución reciente del flujo de inversión extranjera directa ha transitado básicamente por dos etapas. La primera corresponde al notorio impulso que las modificaciones al reglamento de la Ley de Inversión Extranjera de 1993, en el marco de la firma del Tratado de Libre, indujeron en la captación de IED. En esta etapa, y después de atravesar por las perturbaciones ocasionadas por la crisis económica-financiera nacional que detonó en 1994, el flujo de IED creció a un ritmo de 16 por ciento en promedio cada año entre 1994 y 2001, año en que se alcanzó uno de los niveles más elevados ${ }^{3}$ (fig. 1).

Aunque las modificaciones a la Ley de Inversión Extranjera enfocadas a la eliminación de barreras al ingreso de IED al país han continuado hasta la actualidad (Lichtensztejn, 2012), debe mencionarse que después del notorio incremento observado hasta 2001, el flujo de IED ha transitado por una segunda etapa caracterizada por una elevada volatilidad en el corto plazo y también por una tendencia estable en el largo plazo. En este sentido, las fluctuaciones de corto plazo están enmarcadas en la reciente crisis financiera internacional que ocasionaron las disminuciones en el flujo de IED captada en México entre 2008 y 2009; y en concordancia con el entorno internacional de la IED, una nueva caída en 2012. Sin embargo, aunque el flujo de IED en México parece retomar una senda de recuperación a partir de $2013^{4}$, su dinámica

\footnotetext{
2 En el caso de México, Valdivia (2008) analiza la dinámica de transición de la productividad laboral municipal en el Centro de México mediante diferentes metodologías alternativas, una de ellas con base en Cadenas de Markov clásica y espacial.

${ }^{3}$ De acuerdo con Lichtensztejn (2012), el elevado volumen de IED registrado en 2001 está relacionado con la venta de Banamex a Citibank.

${ }^{4}$ La recuperación del flujo de IED captada en México durante 2013 concuerda con la recuperación registrada de la IED global por la UNCTAD (2014), en cuyo reporte se establece una expectativa de recuperación lenta hacia el 2016 con niveles de IED global en 2014, apenas por encima a los registrados en 2013.
} 


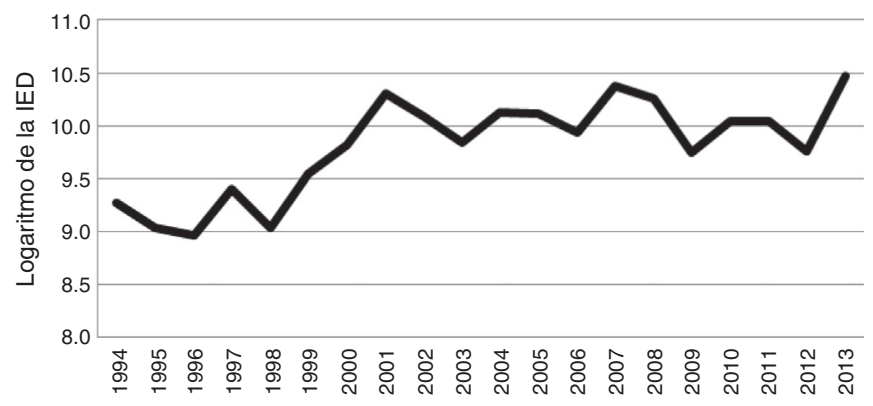

Figura 1. Evolución de la IED en México, 1994-2013.

Fuente: elaboración propia con información del INEGI.

ha sido estable con un modesto crecimiento medio anual de 1.3 por ciento entre 2001 y 2013 (fig. 1).

El esfuerzo gubernamental por atraer mayores flujos de IED al país ha tenido resultados diferenciados, particularmente cuando se observa su evolución en las entidades federativas. Una primera inspección de la IED regional permite apreciar dos rasgos generales de su dinámica: 1) la heterogeneidad regional en la captación de flujos de IED y 2) una tendencia a la divergencia global en la captación de IED regional.

$\mathrm{Al}$ respecto, en el anexo 1 (véanse los anexos) se presenta la inversión extranjera directa acumulada en los periodos 1994-1998 y 2009-2013 con fines comparativos. Ahí se aprecia que una sola entidad, el Distrito Federal, captó consistentemente más del cincuenta por ciento de la IED acumulada en ambos periodos; seguida con una distancia notable por Nuevo León, entidad que logró captar el 10.6 por ciento en el primer periodo, y con una pequeña disminución en su participación, el 8.31 por ciento en el segundo periodo. Algunas entidades han visto reducida su capacidad de atracción notoriamente; por ejemplo, Baja California redujo su participación de 5.48 a 3 por ciento, disminuyendo tres posiciones en ambos periodos. Por su parte, aunque Tamaulipas tuvo un pequeño incremento en la captación acumulada, su participación se redujo de 3.6 a 1.7 por ciento también en ambos periodos. Notablemente, el estado de Hidalgo ha atravesado por un proceso de pérdida de IED que ha ocasionado una importante disminución en su posicionamiento como receptor de la misma.

Es importante mencionar que algunos estados tradicionalmente caracterizados por su baja capacidad para atraer IED, han transitado paulatinamente hacia una mayor captación. Es el caso de los estados ubicados en el sur del país, tales como Chiapas y Oaxaca, cuya captación acumulada de IED se incrementó 48 y 46 veces, respectivamente, entre ambos periodos. Incluso el estado de Guerrero, mejor posicionado que Chiapas y Oaxaca, triplicó la IED acumulada en el mismo lapso de tiempo (anexo 1).

Por otro lado, aunque algunas entidades han avanzado y otras retrocedido en su capacidad para captar IED, la dinámica regional parece acompañarse por un proceso de divergencia global en los flujos de IED que ingresan a las entidades. En la figura 2 se observa que el valor de la mediana del flujo de IED regional ha retomado un desplazamiento ascendente a partir de 2011, colocándose en un nivel mayor que en los primeros años de la década de los noventa, lo cual es consistente con el aumento del flujo agregado de IED observado en la figura 1. Sin embargo, en la figura 2 también se aprecia una creciente dispersión en la IED regional, expresada en el aumento de la altura de las barras a medida que pasa el tiempo. De hecho, la línea oscura que muestra 


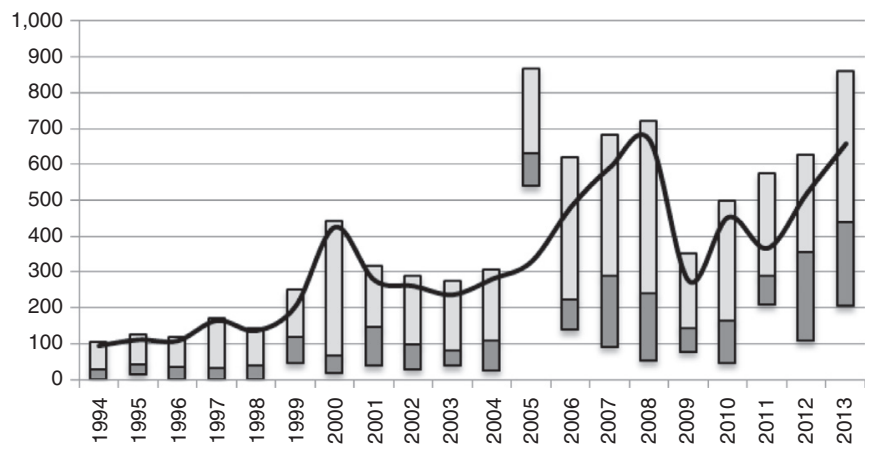

Figura 2. Evolución de la dispersión de la IED regional en México 1994-2013.

La gráfica 2 es similar a una gráfica de caja y bigotes (boxplot). La porción de color gris claro representa la magnitud del cuantil 75 en tanto la porción gris oscuro representa el cuantil 25, y ambas porciones son divididas por la mediana. Se agregó una línea de color oscuro, que representa una medida de dispersión calculada como la diferencia entre la magnitud del cuantil 75 y 25 . Así un aumento de la diferencia implica un aumento de la dispersión y viceversa.

Fuente: elaboración propia con información del INEGI.

la evolución de la diferencia entre la magnitud de los cuantiles 75 y 25, sugiere que si bien la dispersión ha fluctuado, su tendencia es claramente ascendente.

En este sentido, la IED regional se caracteriza por una amplia concentración geográfica, y aunque algunas entidades parecen haber fortalecido su capacidad para atraer IED (y otras se han debilitado), estos esfuerzos parecen inducir un proceso de dispersión regional que se acentúa con el tiempo, particularmente a partir de 2006 cuando la dispersión global se incrementó notablemente en comparación con años anteriores. No obstante, un análisis más completo de la dinámica de la IED regional requiere conocer la transición hacia estadios con niveles mayores (o menores) de captación, considerando inclusive la posible interacción espacial con otras entidades. En particular, el análisis de la dinámica se elabora para el periodo completo desde 1994 hasta 2013 y también para los subperiodos entre los años de 1994 a 2005 y de 2006 a 2013. El análisis por subperiodos permitirá identificar posibles diferencias en la dinámica de transición regional de la IED que pudieran asociarse al aumento en la dispersión global a partir de 2006.

\section{Aspectos metodológicos}

Con la finalidad de analizar la dinámica de la distribución regional de la inversión extranjera directa en México, se recurre al enfoque de cadenas de Markov. A diferencia de los enfoques tradicionales que estudian la dinámica temporal de variables económicas, por ejemplo convergencia sigma o convergencia beta para el caso del ingreso regional, que ofrecen información limitada acerca del comportamiento global de la distribución de variables económicas a través del tiempo (Quah, 1993) e incluso del movimiento relativo de las unidades espaciales dentro de la propia distribución (Rey, 2001), el enfoque de cadenas de Markov ofrece un conjunto de ventajas analíticas relevantes. Por ejemplo, Quah (1996) señala que este enfoque permite estudiar el comportamiento completo de la distribución de las variables económicas a través del tiempo; asimismo, Fingleton (1997) establece que el enfoque de cadenas de Markov permite incorporar choques exógenos y discontinuidades (cambios de régimen) en el análisis. En adición, Rey (2001) sostiene que este enfoque permite incluir la presencia de efectos espaciales, posibilitando así el análisis de la dinámica temporal de la distribución condicionada a la dimensión espacial. 


\section{El enfoque markoviano clásico}

El enfoque markoviano clásico establece que un proceso estocástico tiene la propiedad de Markov si su distribución en un determinado tiempo $t+1$ solamente depende de su distribución inmediata, en t. Esto es, el futuro es explicado únicamente por el presente y no por el pasado. En particular, una cadena de Markov consiste de tres objetos: un estado espacio n-dimensional donde el vector ei toma el valor de 1 en el estado i y cero en el resto de entradas. Una matriz de transición $\mathrm{P}$ de tamaño nxn que registra las probabilidades de moverse de un estado a otro en un periodo; $\mathrm{y}$ un vector $\pi_{0}$ de tamaño $\mathrm{nx} 1$ que indica la probabilidad de encontrarse en el estado i en el periodo inicial 0. En este sentido, de forma resumida los elementos de la matriz de probabilidades de transición P se describen como: $\mathrm{P}_{\mathrm{ij}}=\operatorname{Prob}\left(\mathrm{x}_{\mathrm{t}+1}=\mathrm{e}_{\mathrm{j}} \mid \mathrm{x}_{\mathrm{t}}=\mathrm{e}_{\mathrm{i}}\right)$ (Ljungqvist y Sargent, 2004).

Algunos supuestos relevantes alrededor de los procesos markovianos es que la matriz de transición P es estocástica, es decir, se cumple que la sumatoria de las probabilidades a lo largo de las columnas j para cada renglón i en la matriz de transición P es igual a 1, al igual que ocurre con la sumatoria del vector $\pi_{0 \mathrm{i}}$ a lo largo de i. Asimismo, se supone que la matriz de transición $\mathrm{P}$ es invariante en el tiempo, es decir, se cumple que $\mathrm{p}_{\mathrm{t}, \mathrm{i}, \mathrm{j}}=\mathrm{m}_{\mathrm{t}+\mathrm{b}, \mathrm{i}, \mathrm{j}}$ para todo $\mathrm{b}$.

Es necesario mencionar que el análisis de la dinámica de la IED regional en México se basa particularmente en la matriz de transiciones $\mathrm{P}$, que bajo el supuesto de invariabilidad en el tiempo, presenta dos propiedades relevantes: 1) por un lado, permite conocer el tiempo promedio en el que una región puede transitar de un estado o clase a otro, y 2) a partir del límite de la matriz de transición permite conocer el vector ergódico o de estado estable en el largo plazo. Formalmente, la propiedad de ergodicidad para la matriz $\mathrm{P}$ implica que $\mathrm{P}^{\mathrm{Tb}}=\mathrm{A}$, donde $\mathrm{A}$ es la matriz de estado estable a la que converge y $\mathrm{T}^{\mathrm{b}}$ es el número de años que toma en alcanzarse dicho estado estable (Rey, 2001). En particular, esta última característica permite conocer el equilibrio en el largo plazo al que convergería la distribución de la IED en las entidades federativas de México.

\section{La matriz de transición espacial}

Con la finalidad de incorporar la interacción espacial en el análisis de la dinámica regional de la IED en México, se aplica el enfoque espacial de cadenas de Markov propuesto por Rey (2001). Específicamente, se utiliza la modificación de la matriz de transición clásica $\mathrm{P}$ que condiciona las probabilidades de transición de una región al estado o clase inicial de su rezago espacial, denominada matriz de transición espacial.

Esta versión espacial de la matriz de transición considera la dependencia espacial en forma de rezago espacial, es decir, condiciona la probabilidad de una región específica de transitar hacia un estado o clase, al estado o clase en que las regiones vecinas se encontraban inicialmente. Esta forma de incorporar la interacción espacial al análisis de la dinámica implica que la matriz $\mathrm{P}$ deja de tener dimensión kxk en su versión clásica para tener dimensión kxkxk. De acuerdo con Rey (2001), esta versión espacial permite conocer la influencia que las regiones vecinas tienen en la transición de un estado a otro de una región específica.

Con la finalidad de esclarecer la elaboración e interpretación de la matriz de transición espacial, en la tabla 1 se presenta su organización. Se supone una matriz con k=5 estados o clases definidas como muy bajo (MB), bajo (B), medio (Med), alto (A) y muy alto (MA). Esta definición corresponde al número de clases mutuamente excluyentes calculados mediante cuantiles, y que en este estudio tiene el objetivo de distinguir la capacidad de captación de IED regional a través del tiempo. En particular, la notación $\mathrm{P}_{\mathrm{BB} \mid \mathrm{B}}$ expresa la probabilidad de una región de transitar de un estado o clase bajos (B), hacia otro también bajo (B) en un periodo de tiempo, condicionado 
Tabla 1

Matriz hipotética de transición espacial

\begin{tabular}{|c|c|c|c|c|c|c|}
\hline Rezago espacial & Estado & $\mathrm{MB}$ & B & Med & A & MA \\
\hline \multirow{5}{*}{ MB } & MB & $\mathrm{P}_{\mathrm{MBMB} \mid \mathrm{MB}}$ & $\mathrm{P}_{\mathrm{MBB} \mid \mathrm{MB}}$ & $\mathrm{P}_{\text {MBMed } \mid \mathrm{MB}}$ & $\mathrm{P}_{\mathrm{MBA} \mid \mathrm{MB}}$ & $\mathrm{P}_{\mathrm{MBMA} \mid \mathrm{MB}}$ \\
\hline & B & $\mathrm{P}_{\mathrm{BMB} \mid \mathrm{MB}}$ & $\mathrm{P}_{\mathrm{BB} \mid \mathrm{MB}}$ & $\mathrm{P}_{\mathrm{BMed} \mid \mathrm{MB}}$ & $\mathrm{P}_{\mathrm{BA} \mid \mathrm{MB}}$ & $\mathrm{P}_{\mathrm{BMA} \mid \mathrm{MB}}$ \\
\hline & Med & $\mathrm{P}_{\text {MedMB } \mid \mathrm{MB}}$ & $\mathrm{P}_{\mathrm{MedB} \mid \mathrm{MB}}$ & $\mathrm{P}_{\text {MedMed|MB }}$ & $\mathrm{P}_{\text {MedA|MB }}$ & $\mathrm{P}_{\text {MedMA } \mid \mathrm{MB}}$ \\
\hline & $\mathrm{A}$ & $\mathrm{P}_{\mathrm{AMB} \mid \mathrm{MB}}$ & $\mathrm{P}_{\mathrm{AB} \mid \mathrm{MB}}$ & $\mathrm{P}_{\mathrm{AMed} \mid \mathrm{MB}}$ & $\mathrm{P}_{\mathrm{AA} \mid \mathrm{MB}}$ & $\mathrm{P}_{\mathrm{AMA} \mid \mathrm{MB}}$ \\
\hline & MA & $\mathrm{P}_{\text {MAMB } \mid \mathrm{MB}}$ & $\mathrm{P}_{\mathrm{MAB} \mid \mathrm{MB}}$ & $\mathrm{P}_{\text {MAMed|MB }}$ & $\mathrm{P}_{\mathrm{MAA} \mid \mathrm{MB}}$ & $\mathrm{P}_{\text {MAMA|MB }}$ \\
\hline \multirow{5}{*}{ B } & $\mathrm{MB}$ & $\mathrm{P}_{\text {MBMB } \mid \mathrm{B}}$ & $\mathrm{P}_{\mathrm{MBB} \mid \mathrm{B}}$ & $\mathrm{P}_{\text {MBMed|B }}$ & $\mathrm{P}_{\mathrm{MBA} \mid \mathrm{B}}$ & $\mathrm{P}_{\text {MBMA } \mid \mathrm{B}}$ \\
\hline & $\mathrm{B}$ & $\mathrm{P}_{\mathrm{BMB} \mid \mathrm{B}}$ & $\mathrm{P}_{\mathrm{BB} \mid \mathrm{B}}$ & $\mathrm{P}_{\mathrm{BMed} \mid \mathrm{B}}$ & $\mathrm{P}_{\mathrm{BA} \mid \mathrm{B}}$ & $\mathrm{P}_{\mathrm{BMA} \mid \mathrm{B}}$ \\
\hline & Med & $\mathrm{P}_{\text {MedMB } \mid \mathrm{B}}$ & $\mathrm{P}_{\mathrm{MedB} \mid \mathrm{B}}$ & $\mathrm{P}_{\text {MedMed|B }}$ & $\mathrm{P}_{\text {MedA|B }}$ & $\mathrm{P}_{\text {MedMA } \mid \mathrm{B}}$ \\
\hline & A & $\mathrm{P}_{\mathrm{AMB} \mid \mathrm{B}}$ & $\mathrm{P}_{\mathrm{AB} \mid \mathrm{B}}$ & $\mathrm{P}_{\text {AMed } \mid \mathrm{B}}$ & $\mathrm{P}_{\mathrm{AA} \mid \mathrm{B}}$ & $\mathrm{P}_{\mathrm{AMA} \mid \mathrm{B}}$ \\
\hline & MA & $\mathrm{P}_{\text {MAMB } \mid \mathrm{B}}$ & $\mathrm{P}_{\mathrm{MAB} \mid \mathrm{B}}$ & $\mathrm{P}_{\text {MAMed|B }}$ & $\mathrm{P}_{\mathrm{MAA} \mid \mathrm{B}}$ & $\mathrm{P}_{\text {MAMA|B }}$ \\
\hline \multirow{5}{*}{ Med } & MB & $\mathrm{P}_{\text {MBMB } \mid \text { Med }}$ & $\mathrm{P}_{\mathrm{MBB} \mid \mathrm{Med}}$ & $\mathrm{P}_{\text {MBMed|Med }}$ & $\mathrm{P}_{\mathrm{MBA} \mid \mathrm{Med}}$ & $\mathrm{P}_{\text {MBMA } \mid \text { Med }}$ \\
\hline & $\mathrm{B}$ & $\mathrm{P}_{\mathrm{BMB} \mid \mathrm{Med}}$ & $\mathrm{P}_{\mathrm{BB} \mid \mathrm{Med}}$ & $\mathrm{P}_{\mathrm{BMed} \mid \mathrm{Med}}$ & $\mathrm{P}_{\mathrm{BA} \mid \mathrm{Med}}$ & $\mathrm{P}_{\mathrm{BMA} \mid \mathrm{Med}}$ \\
\hline & Med & $\mathrm{P}_{\text {MedMB } \mid \text { Med }}$ & $\mathrm{P}_{\mathrm{MedB} \mid \mathrm{Med}}$ & $\mathrm{P}_{\text {MedMed|Med }}$ & $\mathrm{P}_{\text {MedA|Med }}$ & $\mathrm{P}_{\text {MedMA|Med }}$ \\
\hline & A & $\mathrm{P}_{\mathrm{AMB} \mid \mathrm{Med}}$ & $\mathrm{P}_{\mathrm{AB} \mid \mathrm{Med}}$ & $\mathrm{P}_{\text {AMed|Med }}$ & $\mathrm{P}_{\mathrm{AA} \mid \mathrm{Med}}$ & $\mathrm{P}_{\mathrm{AMA} \mid \mathrm{Med}}$ \\
\hline & MA & $\mathrm{P}_{\mathrm{MAMB} \mid \mathrm{Med}}$ & $\mathrm{P}_{\mathrm{MAB} \mid \mathrm{Med}}$ & $\mathrm{P}_{\text {MAMed|Med }}$ & $\mathrm{P}_{\text {MAA|Med }}$ & $\mathrm{P}_{\text {MAMA|Med }}$ \\
\hline \multirow{5}{*}{ A } & $\mathrm{MB}$ & $\mathrm{P}_{\mathrm{MBMB} \mid \mathrm{A}}$ & $\mathrm{P}_{\mathrm{MBB} \mid \mathrm{A}}$ & $\mathrm{P}_{\text {MBMed|A }}$ & $\mathrm{P}_{\mathrm{MBA} \mid \mathrm{A}}$ & $\mathrm{P}_{\mathrm{MBMA} \mid \mathrm{A}}$ \\
\hline & $\mathrm{B}$ & $\mathrm{P}_{\mathrm{BMB} \mid \mathrm{A}}$ & $\mathrm{P}_{\mathrm{BB} \mid \mathrm{A}}$ & $\mathrm{P}_{\mathrm{BMed} \mid \mathrm{A}}$ & $\mathrm{P}_{\mathrm{BA} \mid \mathrm{A}}$ & $\mathrm{P}_{\mathrm{BMA} \mid \mathrm{A}}$ \\
\hline & Med & $\mathrm{P}_{\text {MedMB } \mid \mathrm{A}}$ & $\mathrm{P}_{\mathrm{MedB} \mid \mathrm{A}}$ & $\mathrm{P}_{\text {MedMed|A }}$ & $\mathrm{P}_{\text {MedA|A }}$ & $\mathrm{P}_{\text {MedMA } \mid \mathrm{A}}$ \\
\hline & A & $\mathrm{P}_{\mathrm{AMB} \mid \mathrm{A}}$ & $\mathrm{P}_{\mathrm{AB} \mid \mathrm{A}}$ & $\mathrm{P}_{\mathrm{AMed} \mid \mathrm{A}}$ & $\mathrm{P}_{\mathrm{AA} \mid \mathrm{A}}$ & $\mathrm{P}_{\mathrm{AMA} \mid \mathrm{A}}$ \\
\hline & MA & $\mathrm{P}_{\text {MAMB } \mid \mathrm{A}}$ & $\mathrm{P}_{\mathrm{MAB} \mid \mathrm{A}}$ & $\mathrm{P}_{\text {MAMed } \mid \mathrm{A}}$ & $\mathrm{P}_{\mathrm{MAA} \mid \mathrm{A}}$ & $\mathrm{P}_{\text {MAMA|A }}$ \\
\hline \multirow{5}{*}{ MA } & $\mathrm{MB}$ & $\mathrm{P}_{\mathrm{MBMB} \mid \mathrm{MA}}$ & $\mathrm{P}_{\mathrm{MBB} \mid \mathrm{MA}}$ & $\mathrm{P}_{\text {MBMed|MA }}$ & $\mathrm{P}_{\mathrm{MBA} \mid \mathrm{MA}}$ & $\mathrm{P}_{\mathrm{MBMA} \mid \mathrm{MA}}$ \\
\hline & B & $\mathrm{P}_{\mathrm{BMB} \mid \mathrm{MA}}$ & $\mathrm{P}_{\mathrm{BB} \mid \mathrm{MA}}$ & $\mathrm{P}_{\mathrm{BMed} \mid \mathrm{MA}}$ & $\mathrm{P}_{\mathrm{BA} \mid \mathrm{MA}}$ & $\mathrm{P}_{\mathrm{BMA} \mid \mathrm{MA}}$ \\
\hline & Med & $\mathrm{P}_{\mathrm{MedMB} \mid \mathrm{MA}}$ & $\mathrm{P}_{\mathrm{MedB} \mid \mathrm{MA}}$ & $\mathrm{P}_{\text {MedMed|MA }}$ & $\mathrm{P}_{\text {MedA|MA }}$ & $\mathrm{P}_{\text {MedMA|MA }}$ \\
\hline & A & $\mathrm{P}_{\mathrm{AMB} \mid \mathrm{MA}}$ & $\mathrm{P}_{\mathrm{AB} \mid \mathrm{MA}}$ & $\mathrm{P}_{\mathrm{AMed} \mid \mathrm{MA}}$ & $\mathrm{P}_{\mathrm{AA} \mid \mathrm{MA}}$ & $\mathrm{P}_{\mathrm{AMA} \mid \mathrm{MA}}$ \\
\hline & MA & $\mathrm{P}_{\mathrm{MAMB} \mid \mathrm{MA}}$ & $\mathrm{P}_{\mathrm{MAB} \mid \mathrm{MA}}$ & $\mathrm{P}_{\text {MAMed|MA }}$ & $\mathrm{P}_{\mathrm{MAA} \mid \mathrm{MA}}$ & $\mathrm{P}_{\text {MAMA|MA }}$ \\
\hline
\end{tabular}

Fuente: elaboración propia con base en Rey (2001).

a que sus regiones vecinas inicialmente formaban parte de un estado o clase también bajos (B). Similarmente, la notación $\mathrm{P}_{\mathrm{BA} \mid \mathrm{A}}$ indica la probabilidad de una región de transitar de un estado o clase bajos (B) hacia un estado o clase alta (A), condicionado a que interactúa espacialmente con vecinos que se encuentran en el estado o clase alto (A). En este sentido, la matriz de transición espacial expresa con detalle el movimiento de una región hacia estados o clases más altas o bajas a través del tiempo, considerando la influencia favorable o desfavorable de interactuar espacialmente con regiones que se encontraban en estados o clases mayores, inferiores o iguales.

\section{Descripción de las bases de datos}

En el análisis de la dinámica regional de la inversión extranjera directa se utilizan los flujos de IED totales para cada entidad federativa de México con una frecuencia anual de 1994 a 2013, obtenidos del Instituto Nacional de Estadística, Geografía e Informática (INEGI). Siguiendo la estrategia propuesta por Rey (2001), para estimar la matriz de transición espacial se utilizaron los flujos de la IED por entidad relativos respecto al valor medio. Asimismo, el número de entidades federativas y datos temporales permitieron calcular cinco estados o clases mediante cuantiles como sugieren Quah (1993), Rey (2001) y Wren y Jones (2010), de tal forma que cada estado/clase representa la capacidad de captación de IED a la que una entidad federativa puede transitar en un periodo de tiempo. Por su parte, en el cálculo de la matriz de transición espacial, el rezago espacial se obtuvo mediante la elaboración de una matriz de pesos espacial tipo Queen de primer 
Tabla 2

Estadística descriptiva de la IED regional en México (millones de dólares)

\begin{tabular}{lccrrr}
\hline & 1994 & 1998 & 2003 & 2008 & 2013 \\
\hline Media & 332.7 & 261.7 & 590.5 & 885.5 & $1,099.6$ \\
Des. estándar & $1,339.2$ & 720.0 & $2,017.6$ & $2,422.0$ & $3,820.4$ \\
Máximo & $7,602.9$ & $4,000.6$ & $11,441.1$ & $13,753.2$ & $21,882.5$ \\
Mínimo & 0.1 & 0.1 & -11.5 & -17.1 & -136.1 \\
Observaciones & 32 & 32 & 32 & 32 & 32 \\
\hline
\end{tabular}

Fuente: elaboración propia.

Tabla 3

Matriz de transición global probabilística para la IED regional en México, 1994-2013

\begin{tabular}{lllllll}
\hline Estado/cuantil & MB & B & Med & A & MA & VPEE \\
\hline MB & 0.5667 & 0.2833 & 0.0917 & 0.0500 & 0.0083 & 0.1714 \\
B & 0.2632 & 0.3772 & 0.2632 & 0.0877 & 0.0088 & 0.1645 \\
Med & 0.1111 & 0.2393 & 0.3675 & 0.2650 & 0.0171 & 0.1927 \\
A & 0.0351 & 0.0263 & 0.2719 & 0.5088 & 0.1579 & 0.2046 \\
MA & 0.0090 & 0.0090 & 0.0270 & 0.0991 & 0.8559 & 0.2668 \\
\hline
\end{tabular}

Fuente: cálculos propios con información del INEGI.

orden, que captura la interacción espacial de una región con las regiones vecinas inmediatas que tocan cualquier frontera o vértice ${ }^{5}$. Con esta matriz la capacidad de captación de IED de los vecinos adyacentes a una entidad específica se calcula como un promedio ponderado entre la IED de dichos vecinos para posteriormente obtener los cuantiles correspondientes al periodo inicial.

En la tabla 2 se observan algunas características de la IED regional mediante estadísticas básicas. Por ejemplo, el promedio regional se ha incrementado aproximadamente tres veces entre 1994 y 2013; sin embargo, también se ha incrementado la dispersión medida a través de la desviación estándar. Rasgos que confirman el comportamiento de la IED observado en la fig. 2. De hecho, esta dispersión se refleja en en el incremento en términos absolutos para los valores máximo y mínimo de la IED captada por las entidades federativas del país.

\section{Dinámica de la distribución regional de la inversión extranjera directa en México de 1994 a 2013}

En este apartado se analiza la distribución espacio-temporal de la inversión extranjera directa en México para el periodo completo de 1994 a 2013, mediante la matriz de transición de probabilidades (P) descrita en el apartado metodológico. En la tabla 3 se presenta la matriz de transición global probabilística ${ }^{6}$. En esta los elementos en la diagonal principal corresponden a la probabilidad de que una región se mantenga en su clase original sin transitar hacia una clase diferente

\footnotetext{
${ }^{5}$ El análisis de la transición espacial de la IED sigue la propuesta de Rey (2001) de utilizar una matriz de pesos espacial tipo Queen. Formalmente, la matriz W describe la interacción entre una entidad federativa i y las entidades vecinas j donde $\mathrm{j}=1, \ldots, \mathrm{N}$, con $\mathrm{i} \neq \mathrm{j}$, suponiendo que $0 \leq \mathrm{wij} \leq 1$ con wij $=0$ si $\mathrm{i}=\mathrm{j}$. Además se cumple que $\sum$ wij $=1$. Existen otros criterios de contigüidad, sin embargo, la matriz tipo Queen tiene la ventaja de modelar con mayor flexibilidad la interacción espacial cuando una entidad vecina j toca en cualquier frontera o vértice a la entidad i.

${ }^{6}$ La matriz de transición global probabilística se obtiene calculando los cuantiles para la información agrupada a través del tiempo y espacio (pooling).
} 
en un periodo de tiempo, en tanto que los elementos fuera de la diagonal principal reportan la probabilidad que tiene una región de transitar hacia una clase diferente.

En particular, se observa que la probabilidad de que una región preserve su ubicación en su clase original es comparativamente mayor que la probabilidad de transitar hacia una nueva clase, notablemente en las clases MB y MA que ubican las regiones con menor y mayor capacidad de atracción de inversión extranjera, respectivamente. Es decir, una región que se encuentre entre el grupo con menor capacidad de atracción de inversión extranjera directa tiene una probabilidad de 56 por ciento de permanece ahí, mientras que una región ubicada en el nivel más alto de atracción de inversión tiene una probabilidad de 85 por ciento de continuar siendo altamente atractiva para los inversionistas extranjeros. En el resto de clases, la probabilidad de que una región permanezca en su clase/estado original es también elevada, aunque la probabilidad de moverse hacia un nuevo estadio no es despreciable (tabla 3 ).

En este contexto, la distribución espacio-temporal de la inversión extranjera directa en México tiene implicaciones importantes acerca de su dinámica, particularmente, de su movilidad regional. Por ejemplo, el índice de movilidad regional de Shorrocks $(1978)^{7}$ calculado para el periodo de 1994 a 2013 sugiere una movilidad regional moderada (anexo 2). Este panorama global de movilidad regional moderada en los flujos de inversión extranjera directa recoge el hecho señalado anteriormente de que las regiones con mayor y menor capacidad de atracción de inversión extranjera tienen una elevada probabilidad de continuar con esta característica, mientras que las regiones ubicadas en las clases intermedias tienen una probabilidad no despreciable de poder transitar hacia una nueva clase. Este panorama sugiere que la dinámica espacio-temporal de la inversión extranjera en México se caracteriza por un proceso de divergencia regional. De hecho, el vector de probabilidades de estado estable indica que en el largo plazo la distribución espacio-temporal tenderá a ser multimodal, en otras palabras, los flujos de inversión extranjera estarán dispersos en las diferentes clases/estados. En este sentido, no se aprecia un proceso de convergencia hacia la clase intermedia, aunque se observa una probabilidad relativamente mayor a concentrarse en la clase más elevada.

En la tabla 4 se presenta la matriz de transición probabilística de la inversión extranjera directa regional condicionada a la interacción espacial con las regiones vecinas. En el primer bloque se aprecia la dinámica espacio-temporal del flujo de IED de las regiones asociadas espacialmente con regiones vecinas en la clase MB, es decir, con la menor capacidad de atracción de inversión extranjera directa. En particular, se observa que la probabilidad de que una región permanezca en la clase MB es notablemente elevada, con 63 por ciento, si interactúa espacialmente con regiones ubicadas inicialmente en dicha clase MB.

Asimismo, resalta el caso de las regiones ubicadas en el grupo con mayor capacidad de atracción de inversión (MA) que interactúan espacialmente con regiones ubicadas en la clase MB; en esta ocasión, la probabilidad de permanecer en el grupo de las regiones con mayor capacidad de atracción de inversión es de 50 por ciento, con probabilidades del 25 por ciento de descender a clases con menor capacidad de atracción. Este resultado contrasta significativamente con la probabilidad del 85 por ciento de permanecer en el grupo de regiones altamente receptoras observado en la tabla 3, cuando no se condicionó el proceso de transición a la interacción espacial con las regiones vecinas.

\footnotetext{
7 El índice de movilidad propuesto por Shorrocks (1978) ofrece una medida global de la movilidad regional de una variable económica específica. El índice toma valores entre cero y uno, con un valor cercano a uno (cero) indicando una alta (baja) movilidad regional. El cálculo se realiza con base en la fórmula: $m=n-\operatorname{tr}(p) / n-1$ donde n es el número de clases/estados y tr es la traza de la matriz de transición P.
} 
Tabla 4

Matriz de transición probabilística espacialmente condicionada para la IED regional en México, 1994-2013

\begin{tabular}{|c|c|c|c|c|c|c|}
\hline Rezago espacial & Estado/cuantil & MB & $\mathrm{B}$ & Med & A & MA \\
\hline \multirow{5}{*}{ MB } & MB & 0.6364 & 0.2045 & 0.1136 & 0.0455 & 0.0000 \\
\hline & $\mathrm{B}$ & 0.4615 & 0.3077 & 0.2308 & 0.0000 & 0.0000 \\
\hline & Med & 0.1250 & 0.2917 & 0.3750 & 0.2083 & 0.0000 \\
\hline & A & 0.0588 & 0.1176 & 0.1765 & 0.4118 & 0.2353 \\
\hline & MA & 0.0000 & 0.0000 & 0.2500 & 0.2500 & 0.5000 \\
\hline \multirow{5}{*}{$\mathrm{B}$} & MB & 0.5333 & 0.3333 & 0.0667 & 0.0667 & 0.0000 \\
\hline & $\mathrm{B}$ & 0.1364 & 0.3636 & 0.3636 & 0.1364 & 0.0000 \\
\hline & Med & 0.1667 & 0.1667 & 0.2222 & 0.3889 & 0.0556 \\
\hline & A & 0.0000 & 0.0000 & 0.4286 & 0.2857 & 0.2857 \\
\hline & MA & 0.0000 & 0.0286 & 0.0000 & 0.0571 & 0.9143 \\
\hline \multirow{5}{*}{ Med } & MB & 0.6500 & 0.2000 & 0.1500 & 0.0000 & 0.0000 \\
\hline & $\mathrm{B}$ & 0.1481 & 0.5556 & 0.1852 & 0.0741 & 0.0370 \\
\hline & Med & 0.1000 & 0.3667 & 0.2667 & 0.2667 & 0.0000 \\
\hline & A & 0.1111 & 0.0556 & 0.2778 & 0.3333 & 0.2222 \\
\hline & MA & 0.0000 & 0.0000 & 0.0000 & 0.0000 & 1.0000 \\
\hline \multirow{5}{*}{ A } & MB & 0.4444 & 0.3889 & 0.0556 & 0.0556 & 0.0556 \\
\hline & B & 0.3077 & 0.3846 & 0.1923 & 0.1154 & 0.0000 \\
\hline & Med & 0.0455 & 0.0909 & 0.5455 & 0.3182 & 0.0000 \\
\hline & A & 0.0000 & 0.0000 & 0.2143 & 0.6786 & 0.1071 \\
\hline & MA & 0.0625 & 0.0000 & 0.0625 & 0.1250 & 0.7500 \\
\hline \multirow{5}{*}{ MA } & MB & 0.3750 & 0.5000 & 0.0000 & 0.1250 & 0.0000 \\
\hline & B & 0.2308 & 0.1538 & 0.4615 & 0.1538 & 0.0000 \\
\hline & Med & 0.1304 & 0.2174 & 0.4348 & 0.1739 & 0.0435 \\
\hline & A & 0.0270 & 0.0000 & 0.2973 & 0.5946 & 0.0811 \\
\hline & MA & 0 & 0 & 0 & 0.1613 & 0.8387 \\
\hline
\end{tabular}

Fuente: cálculos propios con información del INEGI.

En este sentido, los resultados sugieren no solamente que la inclusión de la interacción espacial en el análisis de la distribución espacio-temporal de la inversión extranjera directa en México ofrece un panorama de la dinámica regional que difiere del análisis no condicionado espacialmente, sino también que la dinámica regional difiere con respecto a la asociación espacial en cada una de las clases.

En particular, en el tabla 4 se aprecia que en el último bloque, la probabilidad de que una región permanezca en el grupo con menor capacidad de atracción de inversión extranjera (clase MB), es de 37 por ciento cuando se considera la interacción con regiones vecinas que pertenecen al grupo con mayor capacidad de atracción de inversión (clase MA), e incluso se aprecia que tiene una probabilidad del 50 por ciento de transitar hacia una clase mayor. Por su parte, la probabilidad de permanecer en el grupo de regiones con mayor capacidad de atracción de inversión extranjera (clase MA) es del 83 por ciento cuando se considera la interacción con regiones vecinas también de la clase MA. Con respecto a las regiones que se encuentran en la clase B tienen una probabilidad de 46 por ciento de transitar hacia la clase Med cuando se considera la interacción con regiones vecinas de la clase MA. Aunque en el caso de las regiones ubicadas en las clases Med y A, las probabilidades de 43 y 59 por ciento respectivamente, sugiere una baja movilidad hacia otras clases.

En este sentido, la dinámica de transición de la IED regional difiere cuando se condiciona a la interacción espacial con regiones que se ubican en las clases con menor o mayor 
Tabla 5

Matriz de probabilidades de estado estable en el largo plazo condicionada espacialmente para la IED regional en México, 1994-2013

\begin{tabular}{llllll}
\hline Estado/cuantil & MB & B & Med & A & MA \\
\hline MB & 0.3756 & 0.2221 & 0.2114 & 0.1299 & 0.0611 \\
B & 0.0753 & 0.0985 & 0.1303 & 0.1411 & 0.5549 \\
Med & 0 & 0 & 0 & 0 & 1 \\
A & 0.0951 & 0.0976 & 0.2535 & 0.3728 & 0.1809 \\
MA & 0.1156 & 0.1346 & 0.2578 & 0.2812 & 0.2108 \\
\hline
\end{tabular}

Fuente: cálculos propios.

capacidad de atracción de inversión. En el caso de la regiones que interactúan con vecinos que son altamente atractivos para la inversionistas extranjeros, se aprecia que las regiones con baja capacidad de atracción (clases MB y B) tienen una probabilidad de transitar hacia clases mayores, y que en las clases mayores (Med, A y MA) la probabilidad de continuar siendo una región atractiva para los inversionistas extranjeros es elevada, particularmente, cuando se encuentran en la clase MA. Este rasgo contrasta con la dinámica observada cuando la interacción ocurre con regiones vecinas que tienen baja capacidad de atracción de inversión, donde las regiones encuentran dificultades para transitar hacia clases/estados más elevados o incluso perder capacidad de atracción de inversión extranjera.

Lo anterior sugiere que las regiones que interactúan con regiones altamente atractivas tienen una probabilidad mayor de incrementar su capacidad de atracción también debido a la presencia de un proceso de difusión espacial favorable. Lo contrario parece ocurrir cuando existe asociación espacial con regiones menos atractivas para la inversión debido a la posible presencia, en este caso, de procesos de difusión espacial desfavorables.

En la tabla 5 se aprecia la distribución espacio-temporal en el estado estacionario para cada una de las matrices de transición condicionadas conforme a la clase/estado correspondiente. La evidencia indica que la dinámica espacio-temporal cuando las regiones interactúan con vecinos poco atractivos para la inversión extranjera (clase MB) tiende hacia un proceso de divergencia, sin que se observe una convergencia hacia la clase intermedia. De hecho, sugiere que en el largo plazo la divergencia se caracteriza por una mayor probabilidad de pertenecer a la clase MB y una baja probabilidad de pertenecer a la clase MA, lo cual no es sorprendente cuando se observa que algunas regiones pueden incluso transitar hacia grupos de regiones con menor capacidad de atracción. En este caso el índice de Shorrocks es el más alto para este bloque en comparación con el resto, y aunque el índice sugiere una movilidad moderada, la distribución en el estado estable implica que no está acompañada por un proceso de convergencia (anexo 2).

Con respecto a las regiones que interactúan con regiones vecinas altamente atractivas para la inversión extranjera (clase MA), la distribución multimodal en el vector de estado estable sugiere la presencia de un proceso de divergencia en el largo plazo, aunque se aprecia una concentración relativamente mayor en las clases Med, A y MA, como resultado de la elevada probabilidad que tienen las regiones ubicadas en las clases MB y B para transitar hacia clases más elevadas. Llama la atención que cuando la interacción espacial ocurre con regiones vecinas ubicadas en la clase intermedia Med, se observa una distribución unimodal ubicada en la clase MA, lo que sugiere un proceso de convergencia regional (tabla 5). 
Tabla 6

Matriz de transición global probabilística para la IED regional en México, 1994-2005

\begin{tabular}{lllllll}
\hline Estado/cuantil & MB & B & Med & A & MA & VPEE \\
\hline MB & 0.6286 & 0.2429 & 0.1143 & 0.0143 & 0.0000 & 0.2188 \\
B & 0.2295 & 0.3770 & 0.2787 & 0.1148 & 0.0000 & 0.1907 \\
Med & 0.1356 & 0.2881 & 0.3051 & 0.2542 & 0.0169 & 0.1844 \\
A & 0.0667 & 0.0667 & 0.2333 & 0.5667 & 0.0667 & 0.1874 \\
MA & 0.0000 & 0.0000 & 0.0286 & 0.0429 & 0.9286 & 0.2174 \\
\hline
\end{tabular}

Fuente: cálculos propios con información del INEGI.

\section{Dinámica de la distribución regional de la inversión extranjera directa en México en el subperiodo de 1994 a 2005}

La dinámica de la distribución espacio-temporal de la inversión extranjera directa en México para el periodo de 1994 a 2005 se caracteriza por una movilidad regional moderada, aunque menor cuando se compara con el periodo completo de análisis; en este periodo, el índice de movilidad regional global de Shorrocks es de 0.54 (anexo 2). En particular, la matriz de transición global de probabilidades de la tabla 5 sugiere que en este periodo la probabilidad de que las regiones ubicadas en los grupos de menor y mayor capacidad de atracción de inversión extranjera (clases MB y MA) preserven su ubicación original es mayor que en el periodo completo de análisis, con 62 y 92 por ciento, respectivamente.

Por su parte, para el resto de regiones ubicadas en las clases B, Med y A, la probabilidad de preservar su ubicación original es mayor que la probabilidad de transitar a nuevos niveles de captación de inversión extranjera. Sin embargo, las probabilidades de transitar hacia una nueva clase no son pequeñas y en algunos casos sugieren una transición hacia clases con menor captación. Por ejemplo, las regiones en la clase Med tienen una probabilidad de 30 por ciento de permanecer en esta clase, aunque también existe una probabilidad de 28 por ciento de transitar hacia la clase $\mathrm{B}$, situación similar para las regiones en la clase A. En este sentido, se justifica la menor movilidad regional que indica el índice de Shorrocks. Por su parte, el vector de probabilidades de estado de estable (VPEE) sugiere que en el largo plazo la distribución espacio-temporal es conducida por un proceso de divergencia con mayor concentración en los extremos de la distribución (clases B y MA) (tabla 6).

Por su parte, el análisis de la matriz de transición cuando se considera la interacción espacial con regiones vecinas sugiere que las regiones asociadas espacialmente con regiones de la clase MB tienen una probabilidad del 80 por ciento de permanecer en la clase MB. Por otro lado, las regiones con una elevada capacidad de atraer inversión extranjera (clase MA) tienen una probabilidad del 40 por ciento de permanecer en esta clase e incluso una probabilidad similar de transitar hacia clases menores. Una situación similar ocurre con las regiones ubicadas en las clases B y A, con una probabilidad mayor de transitar hacia la clase inmediata inferior que de quedarse en su ubicación original. En este caso, la dinámica regional de la inversión extranjera directa es similar a la observada para el periodo completo de análisis, cuando la interacción espacial con regiones vecinas que tienen una baja capacidad para atraer inversión extranjera parece incrementar la probabilidad de permanecer o transitar hacia clases con menor captación (tabla 7).

Lo contrario ocurre cuando las regiones están asociadas espacialmente con regiones vecinas ubicadas en la clase MA. En este caso se observa una movilidad regional moderada, cuando las regiones en la clase MB tienen una probabilidad del 100 por ciento de transitar hacia la clase B, 
Tabla 7

Matriz de transición probabilística espacialmente condicionada para la IED regional en México, 1994-2005

\begin{tabular}{|c|c|c|c|c|c|c|}
\hline Rezago especial & Estado/cuantil & MB & $\mathrm{B}$ & Med & $\mathrm{A}$ & MA \\
\hline \multirow{5}{*}{ MB } & 1 & 0.80 & 0.08 & 0.12 & 0.00 & 0.00 \\
\hline & 2 & 0.50 & 0.25 & 0.13 & 0.13 & 0.00 \\
\hline & 3 & 0.00 & 0.29 & 0.47 & 0.24 & 0.00 \\
\hline & 4 & 0.10 & 0.10 & 0.30 & 0.20 & 0.30 \\
\hline & 5 & 0.00 & 0.00 & 0.20 & 0.40 & 0.40 \\
\hline \multirow{5}{*}{$\mathrm{B}$} & 1 & 0.50 & 0.36 & 0.14 & 0.00 & 0.00 \\
\hline & 2 & 0.07 & 0.36 & 0.43 & 0.14 & 0.00 \\
\hline & 3 & 0.20 & 0.20 & 0.30 & 0.30 & 0.00 \\
\hline & 4 & 0.00 & 0.00 & 0.44 & 0.33 & 0.22 \\
\hline & 5 & 0.00 & 0.00 & 0.00 & 0.07 & 0.93 \\
\hline \multirow{5}{*}{ Med } & 1 & 0.69 & 0.23 & 0.08 & 0.00 & 0.00 \\
\hline & 2 & 0.22 & 0.44 & 0.22 & 0.11 & 0.00 \\
\hline & 3 & 0.08 & 0.42 & 0.17 & 0.25 & 0.08 \\
\hline & 4 & 0.29 & 0.00 & 0.43 & 0.29 & 0.00 \\
\hline & 5 & 0.00 & 0.00 & 0.00 & 0.00 & 1 \\
\hline \multirow{5}{*}{ A } & 1 & 0.50 & 0.33 & 0.17 & 0.00 & 0.00 \\
\hline & 2 & 0.27 & 0.47 & 0.13 & 0.13 & 0.00 \\
\hline & 3 & 0.08 & 0.33 & 0.25 & 0.33 & 0.00 \\
\hline & 4 & 0.05 & 0.11 & 0.11 & 0.68 & 0.05 \\
\hline & 5 & 0 & 0 & 0 & 0 & 1 \\
\hline \multirow{5}{*}{ MA } & 1 & 0 & 1 & 0 & 0 & 0 \\
\hline & 2 & 0.22 & 0.11 & 0.33 & 0.33 & 0 \\
\hline & 3 & 0.09 & 0.18 & 0.55 & 0.18 & 0 \\
\hline & 4 & 0 & 0.05 & 0.15 & 0.75 & 0.05 \\
\hline & 5 & 0 & 0 & 0.05 & 0.05 & 0.90 \\
\hline
\end{tabular}

Fuente: cálculos propios con información del INEGI.

y las regiones en la clase B tiene probabilidades de 33 por ciento de avanzar hacia las clases Med y A, mientras las regiones en las clases Med, A y MA tienen una probabilidad elevada de permanecer en sus ubicaciones originales. En este sentido, se observa que la interacción espacial con regiones altamente atractivas para la inversión extranjera parece favorecer la transición hacia niveles de atracción de inversión mayores en tanto que el proceso contrario estaría ocurriendo cuando la interacción ocurre con regiones de la clase MB, lo que indicaría la presencia de posibles efectos de difusión espacial (tabla 7).

En la tabla 8 se aprecia la distribución espacio-temporal de la inversión extranjera de estado estable. En este caso la evidencia sugiere que la dinámica se acompaña por un proceso de

Tabla 8

Matriz de probabilidades de estado estable en el largo plazo condicionada espacialmente para la IED regional en México, 1994-2005

\begin{tabular}{llllll}
\hline Estado/cuantil & MB & B & Med & A & MA \\
\hline MB & 0.4403 & 0.1520 & 0.2268 & 0.1206 & 0.0603 \\
B & 0.0835 & 0.1002 & 0.1729 & 0.1488 & 0.4945 \\
Med & 0.1556 & 0.1211 & 0.0709 & 0.0446 & 0.6079 \\
A & 0.0863 & 0.1159 & 0.0572 & 0.1152 & 0.6255 \\
MA & 0.0501 & 0.1270 & 0.2402 & 0.3822 & 0.2005 \\
\hline
\end{tabular}

Fuente: cálculos propios. 
Tabla 9

Matriz de transición global probabilística para la IED regional en México, 2006-2013

\begin{tabular}{lllllll}
\hline Estado/cuantil & MB & B & Med & A & MA & VPEE \\
\hline MB & 0.4286 & 0.4048 & 0.0714 & 0.0714 & 0.0238 & 0.2229 \\
B & 0.5278 & 0.3333 & 0.1111 & 0.0278 & 0.0000 & 0.1822 \\
Med & 0.1389 & 0.1111 & 0.4722 & 0.2222 & 0.0556 & 0.1872 \\
A & 0.0000 & 0.0556 & 0.2778 & 0.5000 & 0.1667 & 0.1878 \\
MA & 0.0238 & 0.0000 & 0.0476 & 0.1429 & 0.7857 & 0.2194 \\
\hline
\end{tabular}

Fuente: cálculos propios con información del INEGI.

divergencia debido a que no se observa una tendencia hacia la concentración en las clases intermedias. Pero a medida que la asociación espacial ocurre con regiones altamente atractivas para la inversión extranjera, la probabilidad de pertenecer en el largo plazo a un grupo de regiones con mayor capacidad de atracción también es mayor.

Por ejemplo, las regiones asociadas con vecinos que pertenecen a las clases B, Med y A tienen probabilidades de 49, 60 y 62 por ciento, respectivamente, de transitar hacia el grupo de regiones en la clase MA.

\section{Dinámica de la distribución regional de la inversión extranjera directa en México en el subperiodo de 2006 a 2013}

La dinámica de transición de la inversión extranjera directa regional durante el periodo de 2006 a 2013 comparte rasgos similares con la dinámica del periodo anterior, si bien en la tabla 9 se observan algunas particularidades. Por ejemplo, en este periodo la probabilidad que tiene una región ubicada en la clase $\mathrm{MB}$ de transitar hacia la clase $\mathrm{B}$ es notoriamente mayor, mientras que las regiones ubicadas en la clase B tienen una elevada probabilidad de transitar hacia la clase MB. La probabilidad de permanecer en la clase MA ha disminuido, pero aún es elevada con un 78 por ciento. En este sentido, aunque la dinámica se caracteriza por una movilidad regional mayor como sugiere el índice de Shorrocks (anexo 2), el proceso de divergencia continúa siendo un rasgo común. Al respecto, las probabilidades de estado estable sugieren una distribución multimodal con una concentración un poco mayor en los extremos, alejándose de un proceso de convergencia hacia la clase intermedia.

Con respecto a la transición condicionada a la interacción con las regiones vecinas, se observan también algunas particularidades. En el caso de las regiones que interactúan con vecinos que pertenecen a la clase $\mathrm{MB}$, se aprecia que durante este periodo existe una elevada probabilidad de transitar de la clase MB a la clase B, y con excepción de las regiones ubicadas en la clase B que tienen una alta probabilidad de retroceder hacia la clase MB, sobresale que la probabilidad en la diagonal principal de la matriz global es notablemente alta, lo cual implica que la movilidad hacia niveles grupos de regiones con menor capacidad de atracción es menor en comparación con el periodo de 1994 a 2005 (tabla 10). Sin embargo, en el largo plazo la dinámica sugiere un proceso de divergencia debido a la distribución multimodal, aunque en este periodo la probabilidad de transitar en el largo plazo hacia la clase intermedia es mayor, cuando la interacción ocurre con regiones vecinas en la clase MB (tabla 11). Por otro lado, cuando la interacción ocurre con regiones vecinas ubicadas en la clase MA, es decir, que tienen la mayor captación de inversión extranjera directa, se observa una notable movilidad en las regiones ubicadas en la clase MB (tabla 10), de hecho, el índice de Shorrocks es el mayor con 0.60 (anexo 2). 
Tabla 10

Matriz de transición probabilística espacialmente condicionada para la IED regional en México, 2006-2013

\begin{tabular}{|c|c|c|c|c|c|c|}
\hline Rezago espacial & Estado/cuantil & MB & $\mathrm{B}$ & Med & A & MA \\
\hline \multirow{5}{*}{ MB } & $\mathrm{MB}$ & 0.353 & 0.588 & 0.059 & 0.000 & 0.000 \\
\hline & $\mathrm{B}$ & 0.700 & 0.100 & 0.200 & 0.000 & 0.000 \\
\hline & Med & 0.000 & 0.143 & 0.714 & 0.143 & 0.000 \\
\hline & A & 0.000 & 0.000 & 0.333 & 0.667 & 0.000 \\
\hline & MA & 0.000 & 0.000 & 0.000 & 0.200 & 0.800 \\
\hline \multirow{5}{*}{$\mathrm{B}$} & MB & 0.625 & 0.250 & 0.125 & 0.000 & 0.000 \\
\hline & $\mathrm{B}$ & 0.286 & 0.571 & 0.143 & 0.000 & 0.000 \\
\hline & Med & 0.000 & 0.143 & 0.429 & 0.286 & 0.143 \\
\hline & $\mathrm{A}$ & 0.000 & 0.000 & 0.333 & 0.333 & 0.333 \\
\hline & MA & 0.000 & 0.083 & 0.000 & 0.000 & 0.917 \\
\hline \multirow{5}{*}{ Med } & MB & 0.571 & 0.143 & 0.000 & 0.143 & 0.143 \\
\hline & $\mathrm{B}$ & 0.250 & 0.417 & 0.083 & 0.250 & 0.000 \\
\hline & Med & 0.000 & 0.000 & 0.667 & 0.333 & 0.000 \\
\hline & A & 0.000 & 0.000 & 0.000 & 0.500 & 0.500 \\
\hline & MA & 0.000 & 0.000 & 0.000 & 0.250 & 0.750 \\
\hline \multirow{5}{*}{ A } & MB & 0.333 & 0.500 & 0.000 & 0.167 & 0.000 \\
\hline & B & 0.500 & 0.375 & 0.125 & 0.000 & 0.000 \\
\hline & Med & 0.167 & 0.167 & 0.333 & 0.333 & 0.000 \\
\hline & A & 0.000 & 0.111 & 0.222 & 0.444 & 0.222 \\
\hline & MA & 0.000 & 0.200 & 0.000 & 0.000 & 0.800 \\
\hline \multirow{5}{*}{ MA } & MB & 0.400 & 0.400 & 0.200 & 0.000 & 0.000 \\
\hline & B & 0.000 & 0.000 & 0.000 & 0.000 & 0.000 \\
\hline & Med & 0.286 & 0.143 & 0.286 & 0.143 & 0.143 \\
\hline & A & 0.000 & 0.000 & 0.333 & 0.600 & 0.067 \\
\hline & MA & 0.000 & 0.000 & 0.100 & 0.200 & 0.700 \\
\hline
\end{tabular}

Fuente: cálculos propios con información del INEGI.

En el largo plazo, la dinámica de la inversión extranjera directa se caracteriza por un proceso de divergencia generalizado en el que las regiones que interactúan espacialmente con vecinos ubicados en las clases MB, B y Med tienen una elevada probabilidad de transitar hacia clases mayores en los casos de aquellas, mientras las regiones que interactúan con vecinos en la clases A y MA, tienen una probabilidad importante de transitar hacia una clase menor. Rasgo que contrasta con el proceso de divergencia observado en el periodo anterior, de tal forma que en el primer periodo la divergencia de largo plazo parece ser impulsada principalmente por la transición hacia adelante de las regiones que interactúan con vecinos en la clases B, Med y A, mientras que la

Tabla 11

Matriz de probabilidades de estado estable en el largo plazo condicionada espacialmente para la IED regional en México, 2006-2013

\begin{tabular}{llllll}
\hline Estado/cuantil & MB & B & Med & A & MA \\
\hline MB & 0.229 & 0.212 & 0.391 & 0.168 & 0.000 \\
B & 0.171 & 0.224 & 0.125 & 0.053 & 0.427 \\
Med & 0.000 & 0.000 & 0.000 & 0.333 & 0.667 \\
A & 0.267 & 0.319 & 0.108 & 0.145 & 0.161 \\
MA & 0.133 & 0.096 & 0.235 & 0.279 & 0.256 \\
\hline
\end{tabular}

Fuente: cálculos propios. 
divergencia en el segundo periodo parece ser impulsada por la transición también hacia adelante de regiones que interactúan con vecinos en las clases MB, B y Med.

\section{Conclusiones}

La implementación de reformas regulatorias con la finalidad de motivar el flujo de inversión extranjera directa en el país refleja la importancia que las autoridades gubernamentales han atribuido históricamente a este activo como medio para impulsar el progreso económico nacional. De hecho, diversos estudios nacionales e internacionales han encontrado evidencia empírica que contribuye a fortalecer esta visión de política económica; de tal manera que es previsible que las políticas de fomento a la captación de inversión extranjera directa continúen siendo parte importante de los planes de desarrollo económico en México.

Enmarcadas en la política económica nacional, las entidades federativas en México se han enfrascado en una competencia nacional por atraer la mayor cantidad de flujos de inversión extranjera, con resultados diversos. En este sentido, algunas entidades han tenido mayor éxito que otras, tal es el caso del Distrito Federal y Nuevo León que concentran aproximadamente el 60 por ciento de la inversión extranjera directa acumulada en años recientes. A su vez, algunas entidades como Chiapas, Oaxaca, Tabasco, Nayarit y notablemente Zacatecas, han realizado esfuerzos importantes que han conducido a un incremento en su capacidad de captación. Sin embargo, el panorama en el largo plazo sugiere que bajo las condiciones de capacidad de atracción regional de inversión extranjera directa actuales, la concentración y divergencia regional es un escenario altamente probable.

El análisis de la evolución espacio-temporal de la inversión extranjera directa, mediante el cálculo de la matriz de probabilidades de transición, sugiere que la divergencia regional en la captación de este activo es un proceso que parece afianzarse cuando se analizan diferentes cortes en el tiempo. De hecho, cuando la dinámica se condiciona a la interacción espacial se observa que en el primer periodo de 1994 a 2005 el proceso de divergencia parece ser conducido por una transición hacia clases de mayor captación cuando interactúan con estados ubicados en las clases B, Med y A, en tanto que en el periodo más reciente, de 2006 a 2013, el proceso de divergencia hacia clases mayores estaría impulsado por las regiones que interactúan con vecinos en las clases menores MB, B y Med.

En este contexto, que la dinámica de la inversión extranjera directa se caracterice por un sólido proceso de divergencia regional implica reflexionar acerca del objetivo final de las reformas que promueven la captación de inversión extranjera directa. Parece claro que las reformas y la política económica derivada que promueve la captación de inversión extranjera deben acompañarse no solamente por una visión sectorial sino también por una regional que promueva la creación de incentivos y condiciones para motivar la localización de la inversión extranjera en regiones con limitaciones para capturarla, con el objetivo de aminorar el proceso de divergencia en la captación de inversión extranjera y fomentar un progreso económico regional menos desigual.

\section{Agradecimientos}

Los autores agradecen las observaciones de dos dictaminadores anónimos, útiles en la mejora sustantiva del documento. 


\section{Anexos.}

Tabla A1

Distribución porcentual de la IED por entidad en México

\begin{tabular}{|c|c|c|c|c|c|c|c|}
\hline Posición & Entidad & $\begin{array}{l}\text { Total } \\
\text { acumulado } \\
\text { 1994-1998 }\end{array}$ & Porcentaje & Posición & Entidad & $\begin{array}{l}\text { Total } \\
\text { acumulado } \\
\text { 2009-2013 }\end{array}$ & Porcentaje \\
\hline & & $47,388.5$ & 100 & & & $115,503.89$ & 100 \\
\hline 1 & Distrito Federal & $27,510.7$ & 58.05 & 1 & Distrito Federal & $60,196.92$ & 52.12 \\
\hline 2 & Nuevo León & $5,044.6$ & 10.65 & 2 & Nuevo León & $9,596.29$ & 8.31 \\
\hline 3 & Baja California & $2,598.5$ & 5.48 & 3 & Chihuahua & $6,655.76$ & 5.76 \\
\hline 4 & Chihuahua & $2,497.8$ & 5.27 & 4 & $\begin{array}{l}\text { Estado de } \\
\text { México }\end{array}$ & $6,116.42$ & 5.3 \\
\hline 5 & $\begin{array}{l}\text { Estado de } \\
\text { México }\end{array}$ & $2,401.2$ & 5.07 & 5 & Jalisco & $5,135.2$ & 4.45 \\
\hline 6 & Tamaulipas & $1,719.8$ & 3.63 & 6 & Baja California & $3,508.16$ & 3.04 \\
\hline 7 & Jalisco & 928.9 & 1.96 & 7 & Puebla & $2,739.01$ & 2.37 \\
\hline 8 & Sonora & 701.6 & 1.48 & 8 & Querétaro & $2,664.2$ & 2.31 \\
\hline 9 & Coahuila & 619.3 & 1.31 & 9 & Guanajuato & $1,988.58$ & 1.72 \\
\hline 10 & Puebla & 512.2 & 1.08 & 10 & Aguascalientes & $1,959.82$ & 1.7 \\
\hline 11 & Querétaro & 451.2 & 0.95 & 11 & BC Sur & $1,954.75$ & 1.69 \\
\hline 12 & Quintana Roo & 261.7 & 0.55 & 12 & Tamaulipas & $1,944.19$ & 1.68 \\
\hline 13 & SL Potosí & 246.4 & 0.52 & 13 & Coahuila & $1,931.09$ & 1.67 \\
\hline 14 & Morelos & 226.4 & 0.48 & 14 & Quintana Roo & $1,482.22$ & 1.28 \\
\hline 15 & Durango & 219.8 & 0.46 & 15 & Zacatecas & $1,354.83$ & 1.17 \\
\hline 16 & Sinaloa & 218.6 & 0.46 & 16 & Durango & $1,214.81$ & 1.05 \\
\hline 17 & Aguascalientes & 177.6 & 0.37 & 17 & SL Potosí & $1,089.94$ & 0.94 \\
\hline 18 & Yucatán & 161.7 & 0.34 & 18 & Sinaloa & 757.73 & 0.66 \\
\hline 19 & BC Sur & 153.1 & 0.32 & 19 & Sonora & 689.85 & 0.6 \\
\hline 20 & Hidalgo & 118.6 & 0.25 & 20 & Nayarit & 495.2 & 0.43 \\
\hline 21 & Colima & 117.5 & 0.25 & 21 & Veracruz & 366.87 & 0.32 \\
\hline 22 & Guanajuato & 95.1 & 0.2 & 22 & Tabasco & 305.11 & 0.26 \\
\hline 23 & Veracruz & 91.5 & 0.19 & 23 & Guerrero & 289.63 & 0.25 \\
\hline 24 & Guerrero & 86.4 & 0.18 & 24 & Michoacán & 242.48 & 0.21 \\
\hline 25 & Michoacán & 66.8 & 0.14 & 25 & Oaxaca & 229.36 & 0.2 \\
\hline 26 & Zacatecas & 64.3 & 0.14 & 26 & Tlaxcala & 201.35 & 0.17 \\
\hline 27 & Tlaxcala & 50.5 & 0.11 & 27 & Yucatán & 162.56 & 0.14 \\
\hline 28 & Nayarit & 24.9 & 0.05 & 28 & Chiapas & 126.04 & 0.11 \\
\hline 29 & Tabasco & 9.8 & 0.02 & 29 & Colima & 109.73 & 0.1 \\
\hline 30 & Oaxaca & 4.9 & 0.01 & 30 & Morelos & 91.36 & 0.08 \\
\hline 31 & Campeche & 4.5 & 0.01 & 31 & Campeche & -43.96 & -0.04 \\
\hline 32 & Chiapas & 2.6 & 0.01 & 32 & Hidalgo & -51.6 & -0.04 \\
\hline
\end{tabular}

Fuente: elaboración propia. 
Tabla A2

Movilidad regional mediante el Índice de Shorrocks

\begin{tabular}{llll}
\hline Matriz de transición & $\begin{array}{l}\text { Valor del índice } \\
1994-2013\end{array}$ & $\begin{array}{l}\text { Valor del índice } \\
1994-2005\end{array}$ & $\begin{array}{l}\text { Valor del índice } \\
2006-2013\end{array}$ \\
\hline Matriz no condicionada & 0.581 & 0.549 & 0.594 \\
Matriz de transición espacial & & & 0.473 \\
Vecino en primer cuantil & 0.554 & 0.576 & 0.425 \\
Vecino en segundo cuantil & 0.536 & 0.515 & 0.419 \\
Vecino en tercer cuantil & 0.439 & 0.482 & 0.543 \\
Vecino en cuarto cuantil & 0.439 & 0.420 & 0.603 \\
Vecino en quinto cuantil & 0.521 & 0.538 & \\
\hline
\end{tabular}

Fuente: cálculos propios.

\section{Referencias}

Airola, J. (2008). A regional analysis of the impact of trade and foreign direct investment on wages in Mexico, 1984-2000. Review of Development Economics, 12(2), 276-290. http://dx.doi.org/10.1111/j.1467-9361.2008.00428.x

Carrillo, J. (2000). Las maquiladoras de exportación en México: evolución industrial, aglomeraciones y seguridad e higiene. Documento preparado para el Proyecto de Mejoramiento de las Condiciones de Empleo en la Industria Maquiladora financiado por la STyPS.

Carrillo, J. (2001). Maquiladoras de exportación y la formación de empresas mexicanas exitosas. En E. Dussel (Ed.), Claroscuros, integración exitosa de las pequeñas y medianas empresas en México.. México: CEPAL y editorial JUS, 2001.

Cuevas, A., Messmacher, M. y Werner, A. (2005). Foreign direct investment in Mexico since de approval of NAFTA. The World Bank Economic Review, 19(3), 473-488.

De la Cruz, J., Canfield, C. y González, P. (2009). Economic growth, foreign direct investment and international trade: evidence on causality in the Mexican economy. Revista Brasileira de Economia de Empresas, 9(1), 1-17.

Díaz-Bautista, A. (2006). Foreign direct investment and regional economic growth considering the distance to the northern border of Mexico. Análisis Económico, XXI(46), 355-367.

Domínguez, L. y Brown, F. (2004). Inversión extranjera directa y capacidades tecnológicas. pp. 1-63. CEPAL.

Dussel, E. (2000). La inversión extranjera en México. pp. 1-103. Chile: Serie desarrollo productivo CEPAL.

Dussel, E., Galindo, M., Loría, E. y Mortimore, M. (2007). Inversión extranjera directa en México: desempeño y potencial. México: Siglo XXI, UNAM y Secretaría de Economía.

Escobar, O. (2013). Foreign direct investment (FDI) determinants and spatial spillovers across Mexico's states. The Journal of International Trade and Economic Development, 22(7), 993-1012. http://dx.doi.org/10.1080/ 09638199.2011.624190

Ernst, C., Berg, J. y Auer, P. (2007). Retos en materia de empleo y respuestas de política en Argentina, Brasil y México. Revista de la CEPAL, 91, 95-110.

Fingleton, B. (1997). Specification and testing of Markov chain models: an application to convergence in the European Union. Oxford Bulletin of Economics and Statistics, 59(3), 385-403. http://dx.doi.org/10.1111/1468-0084.00072

García, R. (2001). La maquila y la inversión extranjera directa en México. ICE: Revista de Economía no, 795, 127-140.

Juárez, C. y Ángeles, G. (2013). Foreign direct investment in Mexico. Determinants and its effects on income inequality. Contabilidad y Administración, 58(4), 201-222. http://dx.doi.org/10.1016/S0186-1042(13)71239-7

Kumar, N. (2003). Use and effectiveness of performance requirements: What can be learnt from the experiences of developed and developing countries? En UNCTAD Secretariat (2003). The development dimensión of FDI: policy and rule-making perspectives. New York and Geneva: United Nations.

Lichtensztejn, S. (2012). La inversión extranjera directa en México 1980-2011. México: Universidad Veracruzana.

Ljungqvist, L. y Sargent, T. (2004). Recursive macroeconomic theory. US: The MIT Press.

Mejía, P. (2005). La inversión extranjera directa en los estados de México: evolución reciente y retos futuros. Economía, Sociedad y Territorio Edición especial, (99), 185-236.

Mendoza, J. (2011). Impacto de la inversión extranjera directa en el crecimiento manufacturero de México. Problemas del desarrollo. Revista Latinoamericana de economía, 167(42), 45-69. 
Oladipo, O. (2007). Foreign direct investment and growth in Mexico: an empirical investigation. Proceedings of the Northeast Business and Economics Association, 32, 87-91.

Oladipo, O. y Vázquez, B. (2009). The controversy about foreign direct investment as a source of growth for the Mexican economy. Problemas del desarrollo. Revista Latinoamericana de economía, 40(158), 91-112.

Quah, D. (1993). Empirical cross-section dynamics in economic growth. European Economic Review, 37(2), 426-434.

Quah, D. (1996). Empirics for economic growth and convergence. European Economic Review, 40(6), 1353-1375. http://dx.doi.org/10.1016/0014-2921(95)00051-8

Rey, S. (2001). Spatial empirics for economic growth and convergence. Geographical Analysis, 33(3), $195-214$. http://dx.doi.org/10.1111/j.1538-4632.2001.tb00444.x

Romo, D. (2003). Derramas tecnológicas de la inversión extranjera en la industria mexicana. Comercio Exterior, 53(3), 230-243.

Romero, J. (2012). Inversión extranjera directa y crecimiento económico en México, 1940-2011. Investigación Económica, LXXI(282), 109-147.

Romero, J. (2014). Los límites al crecimiento económico de México. México: El Colegio de México.

Shorrocks, A. (1978). The measurement of mobility. Econometrica, 46(5), 1013-1024.

UNCTAD Secretariat. (2003). The development dimensión of FDI: policy and rule-making perspectives. New York and Geneva: United Nations.

UNCTAD. (2013). World investment report. Global value chains: investment and trade for development. New York and Geneva: United Nations.

UNCTAD. (2014). World investment report. Investing in the SDGs: an action plan. New York and Geneva: United Nations.

Valdivia, M. (2008). Desigualdad regional en el centro de México. pp. 5-34. Una exploración espacial de la productividad en el nivel municipal durante el periodo 1988. 2003 (13) Investigaciones regionales No.

Vera-Cruz, A., Dutrénit, G. y Gil, J. (2005). Derramas de la maquila en un sector de pequeñas y medianas empresas proveedoras. Comercio Exterior, 55(11), 970-986.

Vergara, R., de Jesús, L. y Carbajal, Y. (2015). IED y empleo en la región norte de México, 2004.1-2013.4 Un análisis para el sector industrial. Equilibrio Económico, II(1), 65-94.

Waldkirch, A. (2010). The effects of foreign direct investment in Mexico since NAFTA. The World Economy, 33, 710-745. http://dx.doi.org/10.1111/j.1467-9701.2009.01244.x

Wren, C. y Jones, J. (2010). FDI location across British regions and agglomerative forces: a Markov analysis. Spatial Economic Analysis, 7(2), 265-286. http://dx.doi.org/10.1080/17421772.2012.669492 